\title{
The use of faces as stimuli in neuroimaging and psychological experiments: A procedure to standardize stimulus features
}

\author{
Ed H. B. M. Gronenschild, Floortje Smeets, Eric F. P. M. Vuurman, \\ MaRTin P. J. van BOXTEL, AND JELle JolleS \\ Maastricht University, Maastricht, The Netherlands
}

\begin{abstract}
In psychological experiments involving facial stimuli, it is of great importance that the basic perceptual or psychological characteristics that are investigated are not confounded by factors such as brightness and contrast, head size, hair cut and color, skin color, and the presence of glasses and earrings. Standardization of facial stimulus materials reduces the effect of these confounding factors. We therefore employed a set of basic image processing techniques to deal with this issue. The processed images depict the faces in grayscale, all at the same size, brightness, and contrast, and confined to an oval mask revealing only the basic features such as the eyes, nose, and mouth. The standardization was successfully applied to four different face databases, consisting of male and female faces and including neutral as well as happy facial expressions. An important advantage of the proposed standardization is that featural as well as configurational information is retained. We also consider the procedure to be a major contribution to the development of a de facto standard for the use of facial stimuli in psychological experiments. Such methodological standardization would allow a better comparison of the results of these studies.
\end{abstract}

In psychological experiments, visual stimuli are often used in experiments addressing a wide variety of scientific questions. In terms of object categories, faces are of particular importance, since they are highly significant for humans and are processed faster and differently than other object types (Bruce, Doyle, Dench, \& Burton, 1991; Tanaka \& Farah, 1993; Yin, 1969). A specific area in the brain, called the fusiform face area (FFA), located in the fusiform gyrus, is known to be active only when people are watching images of faces (e.g., Kanwisher, McDermott, \& Chun, 1997).

Experiments employing facial stimuli can differ widely in nature. For example, some researchers have studied the developmental changes of face processing in children (e.g., Aylward et al., 2005), whereas others have addressed the question of how emotional expressions are recognized and processed by normal and disturbed individuals (e.g., Surguladze et al., 2006). The difference in processing of familiar and unfamiliar faces was investigated by, among others, Dubois et al. (1999), and the effect of features or feature relationships on face perception was reported by, for example, Buchala, Davey, Frank, Loomes, and Gale (2005). A common factor in these studies was the presentation of facial stimuli to the participants. However, a comparison among the studies of the mode of presentation and the distinctive features of these stimuli reveals large and fundamental differences. Both physical features, such as contrast, luminance, and saturation, as well as content fea- tures, such as face geometry and size, are either not fully described or are vastly different. This heterogeneity in facial stimuli makes the outcomes of such studies difficult or even impossible to compare. Furthermore, standardization of stimulus content within studies may be imperative when, for example, facial comparison is addressed.

In face recognition, two types of information are important: (1) featural and (2) configurational information (e.g., spatial relationships between features and feature proportions [Rakover, 2002]). In this respect, the most important features are the nose, mouth, and eyes and their spatial relationships (Tanaka \& Farah, 1993). For instance, in the experiments reported by Barton, Keenan, and Bass (2001) it was found that inversion of the mouth significantly impaired face recognition and that inversion of the eyes slightly impaired face recognition. In addition, even schematic faces, represented by only two circles for the eyes, a vertical elliptical shape for the nose, and a horizontal elliptical shape for the mouth activate the FFA (Tong, Nakayama, Moscovitch, Weinrib, \& Kanwisher, 2000). Cropping the image to an oval shape that reveals only these features ensures that face recognition concentrates on these essential facial features. Moreover, a more or less oval mask has proven to be appropriate in previous research (e.g., Bokde et al., 2005; Leinsinger et al., 2007; White \& $\mathrm{Li}, 2006$ ).

In preparation for an fMRI study focusing on face matching, we set out to construct a set of facial stimuli

E. H. B. M. Gronenschild, ed.gronenschild@np.unimaas.nl 
that were equal with regard to very basic features of the face such as head size, eye-nose-mouth geometry, hair cut, hair color, facial expression, and so on. These stimuli were used in an experiment investigating basic processes of face recognition, in which participants were presented pairs of either blurred or nonblurred faces simultaneously for $2.8 \mathrm{sec}$ and had to respond by pressing a button if they considered the two faces equal. When these features are very distinctive (e.g., glasses vs. no glasses), basic face matching processes are severely confounded, because attention is drawn to distinguishing isolated characteristics of the face or external features such as hair.

Our aim was to develop a robust procedure to standardize facial stimuli in order to ensure that face recognition is based on pattern identification in the eyes-mouth triangle and not on isolation (extraction) of salient features. A potential advantage is that, when this standardization is more widely adopted, the results of different facial perception studies can be compared more easily. In this article, we report on our technique to accomplish this standardization and its application to a number of face databases.

\section{METHOD}

In this study, examples from the Extended Multimodal Face Database (XM2VTSDB) (Messer, Matas, Kittles, Luettin, \& Maitre, 1999) were used as a starting point for the development of our technique. The database contains studio shots in full color of natural-looking faces of both males and females placed in front of a uniform background and measuring $720 \times 576$ pixels in size. Only shots of faces in a frontal orientation were selected for our experiment. Our goal was to convert each image into a grayscale image that was limited to an oval "window" of the original face. This oval should include the mouth, nose, and eyes, of which the relative positions were to be standardized. All distinguishing features such as hair style and color, clothing, presence or absence of ears, glasses, and earrings, and so on were to be removed.

To realize this, we found that the position and geometry of the eyes and mouth provided sufficient information. For this particular database, we developed an algorithm that automatically detected the eyes and mouth. Only a few images in the database required manual preprocessing to enable a successful automatic detection. The actual processing involved manipulation of the images in a few distinctive steps. These steps followed well-established image processing techniques widely used in psychophysical studies, among others. They were, however, adapted for the particular type of stimuli in the present procedure. The three basic steps involved were:

1. Manual preprocessing: removal of disturbing features such as glasses, earrings, and so on as preliminary steps for the automatic detection of the eyes and mouth.

2. Geometrical standardization: scaling of each face to a fixed size and confinement to an oval shape on the basis of the positions of the eyes and mouth.

3. Optical standardization: conversion to a fixed level of contrast.
In the following, we will describe each step in more detail.

\section{Manual Preprocessing}

All manual processing was performed with Adobe Photoshop. Typical examples were (1) removal of glasses, also involving adjustments to the color of the skin around the eyes due to shadow artifacts and sometimes due to opacity effects of the lenses; (2) removal of hair on the forehead or artificially extending the forehead in order to produce enough bare skin above the eyes; (3) removal of earrings. An additional uncovering or revealing of the ears was not considered necessary in view of the limited area of the oval mask in which the faces were presented (see below). Finally, if necessary, a face was put in an upright position in order to eliminate the pose as a possible confounding factor. In total, 255 out of 295 faces were considered suitable for our study, of which about 40 were manually edited.

\section{Geometrical Standardization}

The geometrical standardization implied that all faces were centered and scaled to the same size. Because the scaling depended on the distance between both eyes and between the mouth and eyes, we developed an algorithm that fully automatically detects the eyes and mouth in an image. Since this algorithm is rather heuristic and is tailored to the XM2VTSDB, we will only briefly describe it here. The face presented in Figure 1A serves as an example.

A preliminary step involved the extraction of a region of interest (ROI) called the "head mask." For this purpose we used the hue image, because we noticed that the largest contrast between the face and its surroundings was present in the hue component of the hue, saturation, and value (HSV) color space. This was followed by a simple thresholding technique, after which a smoothing by means of a morphologic opening filter was applied (Haralick, Sternberg, \& Zhuang, 1987). This produced a clean head mask that was used in the subsequent processing steps (Figure 1B).

For the detection of the eyes, a technique called "unsharp masking" was employed (Gonzalez \& Woods, 1992): From the original, now grayscale image, a somewhat blurred image was subtracted. For blurring, we used neighborhood averaging, whereby the grayscale value of a pixel was substituted by the average grayscale value within a block of $5 \times 5$ surrounding pixels. The result of the subtraction was an image in which only fine-scale structures pop up (Figure 1C). Of particular interest are the two dots present in both eyes as a result of the use of two studio lights. We observed that in most cases these dots were the brightest objects in the filtered image. For the final position of each eye, we took the average of the positions of the two reflection dots in each eye.

The mouth was detected by means of high-pass filtering of the grayscale image. In this case we used a Prewitt operator (Gonzalez \& Woods, 1992), also called "edge tracing," to derive the edges or grayscale gradients in the image (Figure 1D). Not only the eyes but also the mouth is striking in this image. Within a rectangular ROI, the 

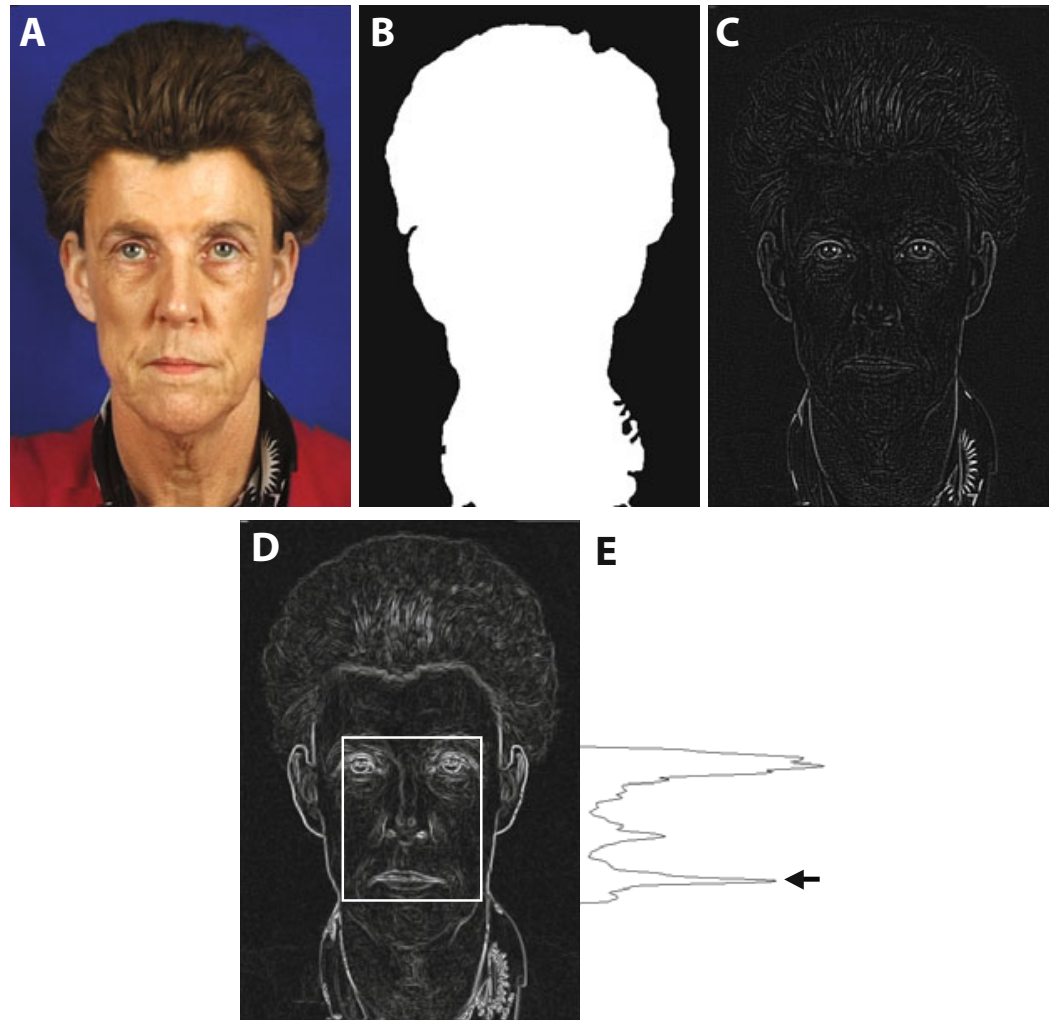

Figure 1. The image processing steps for the automatic detection of the eyes and mouth in the color image shown in panel A. From the color image, the hue image was derived, which was thresholded and morphologically smoothed to obtain the head mask depicted in panel B. Unsharp masking was applied to the color image converted to grayscale (C). In this image, only fine-scale structures pop up. The two dots in each eye, caused by the reflection of the two studio lights used, are the brightest objects in the image and can be detected easily. For the position of each of the eyes, the mean position of the two dots was taken. For the detection of the mouth, high-pass filtering was used by applying a Prewitt operator. This produced an image in which the gradients or edges were visible (D). Summation of the grayscale values along horizontal lines confined to the rectangular region of interest (ROI) generates the profile shown in panel E. A few peaks can be identified, corresponding to the eyes, nostrils, and mouth. For the vertical position of the mouth, we took the position of the peak pointed to by the arrow. The horizontal position was set equal to the position halfway between the eyes.

gradient values in the filtered image were summed along horizontal lines. The result was a profile in which a few peaks were discernible: The upper peak was produced by the eyes, the second peak from above was caused by the nostrils, and the lower peak was from the mouth (Figure 1E). Note that with this procedure, only the vertical position of the mouth was derived; its horizontal position was set equal to the position halfway between both eyes. The rectangular ROI was defined to include the eyes and mouth and to be located inside the head mask. Its position and extent were derived experimentally.

For the intended study we decided to present the faces only partially - that is, within an oval shape, the most prevalent outline of the human head. The definition of the oval mask was rather straightforward. A circle was constructed through the positions of the eyes and mouth. This circle was then magnified horizontally by a factor of 1.3 and vertically by a factor of 1.6 (Figure 2). These factors were experimentally derived on the basis of the following considerations: (1) If the oval mask were too small, too little of the face would be presented to make recognition possible; (2) if the oval mask were too wide, ears could be included for some of the faces, adversely affecting face matching; (3) if the oval mask were too long, the chin could be included for some of the faces, with similar adverse effects on the face matching task. The first condition would lead to extreme task difficulty or even an impossible task, whereas the other two conditions would have just the opposite effect because matching would be based on one or more outstanding features (e.g., chin vs. no chin) instead of an analysis of a face as a whole.

Of all the 255 suitable faces present in the XM2VTSDB, the oval mask was derived and subsequently its average size (width and height). Finally, all faces were linearly scaled, independently in $x$ and $y$ directions, so that each scaled oval mask size equaled the average size. This scal- 


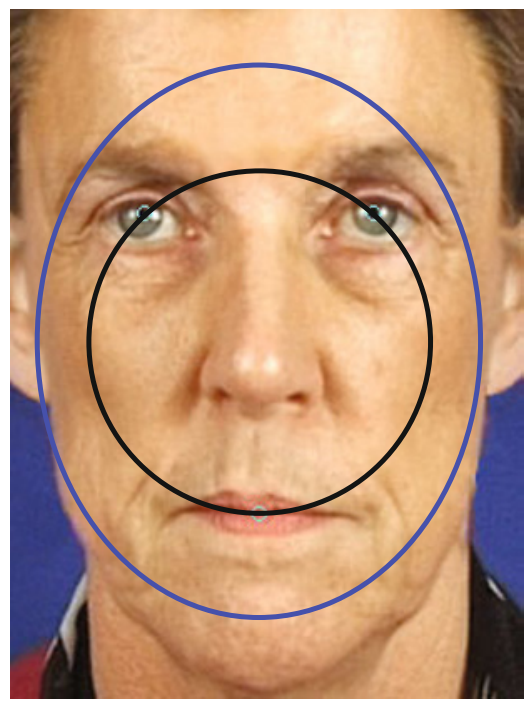

Figure 2. The definition of the oval mask. Through the detected positions of the eyes and mouth (indicated by small circles), a circle was constructed. This circle was then magnified horizontally by a factor of 1.3 and vertically by a factor of 1.6 .

ing technique bears some similarity to that proposed by Dalal and Phadke (2007). Although our technique is less sophisticated, in that only 3 landmarks are used, rather than 16, it is accurate enough for the intended purpose.

The faces were centered by extracting only the part within the oval mask. After conversion to grayscale to control for color variations between images, the geometri- cal standardization of the face was completed by putting a rectangular and black frame around the oval mask with dimensions 30 pixels larger than the oval mask's width and height, respectively. The final images measured $220 \times$ 256 pixels.

\section{Optical Standardization}

Another factor that affects perception and thus face matching (or stimuli processing) is the grayscale contrast of the faces. This can vary from face to face due to changing illumination conditions or camera exposure settings. A standardization is required to reduce this kind of variation. An almost infinite number of techniques exists to manipulate contrast in both linear and nonlinear ways. We decided to use a linear mapping of the grayscale values onto the range of grayscale values available on a computer monitor (range 0-255). A few possible mappings were considered. For this purpose, the image histogram of the oval face image was derived, from which a few features were extracted, such as the minimum, maximum, and mean grayscale values. In addition, a so-called " $x \%$ threshold" was computed - that is, $x \%$ of the pixels have a grayscale value below this threshold. Examples of the investigated mappings are depicted in Figure 3. For the original image (Figure 3A), the minimum and maximum grayscale values were 7 and 221, respectively. Mapping the range 7-221 onto 0-255 improved the contrast considerably (Figure 3B). We further enhanced the contrast by taking a range between the minimum grayscale value and the $98 \%$ threshold, corresponding to 202 (Figure 3C). We obtained a third mapping by taking a range between

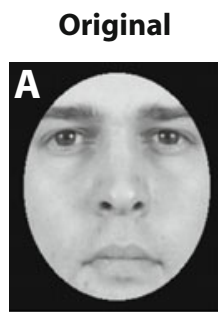

$0-255$
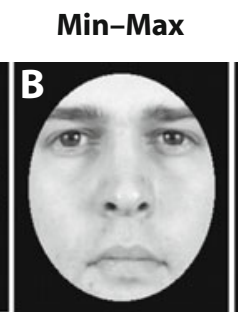

$7-221$
Min-98\%

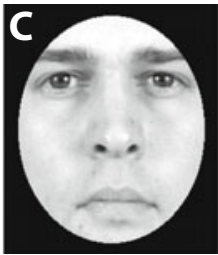

7-202
$1 \%-99 \%$

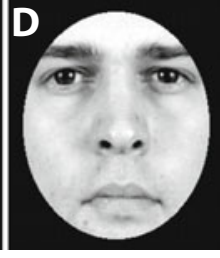

38-204

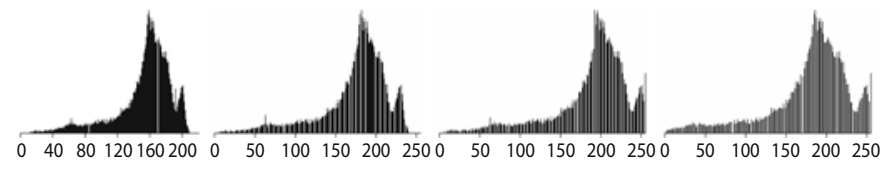

Figure 3. The optical standardization. A few possible linear mappings (contrast manipulations) of grayscale values onto the available range of 0 to 255 are illustrated. The upper panel shows the images, whereas the corresponding image histograms for pixels within the oval mask are depicted in the lower panel. The original image without contrast enhancement is shown in panel A. The contrast is improved if the mapping is from the minimum grayscale value $(=7)$ to the maximum $(=221)(B)$. Another enhancement occurs if, instead of the maximum, the $98 \%$ threshold is taken (C). This threshold is equal to 202 and means that $\mathbf{9 8 \%}$ of the pixels have a grayscale value below 202 . The last manipulation considered by us is a mapping from the $1 \%$ threshold $(=38)$ to the $99 \%$ threshold $(=204)$, as shown in panel $D$. We note that in all cases the histogram is stretched by the mappings and that nonoccupied grayscale values arise due to rounding-off effects. 

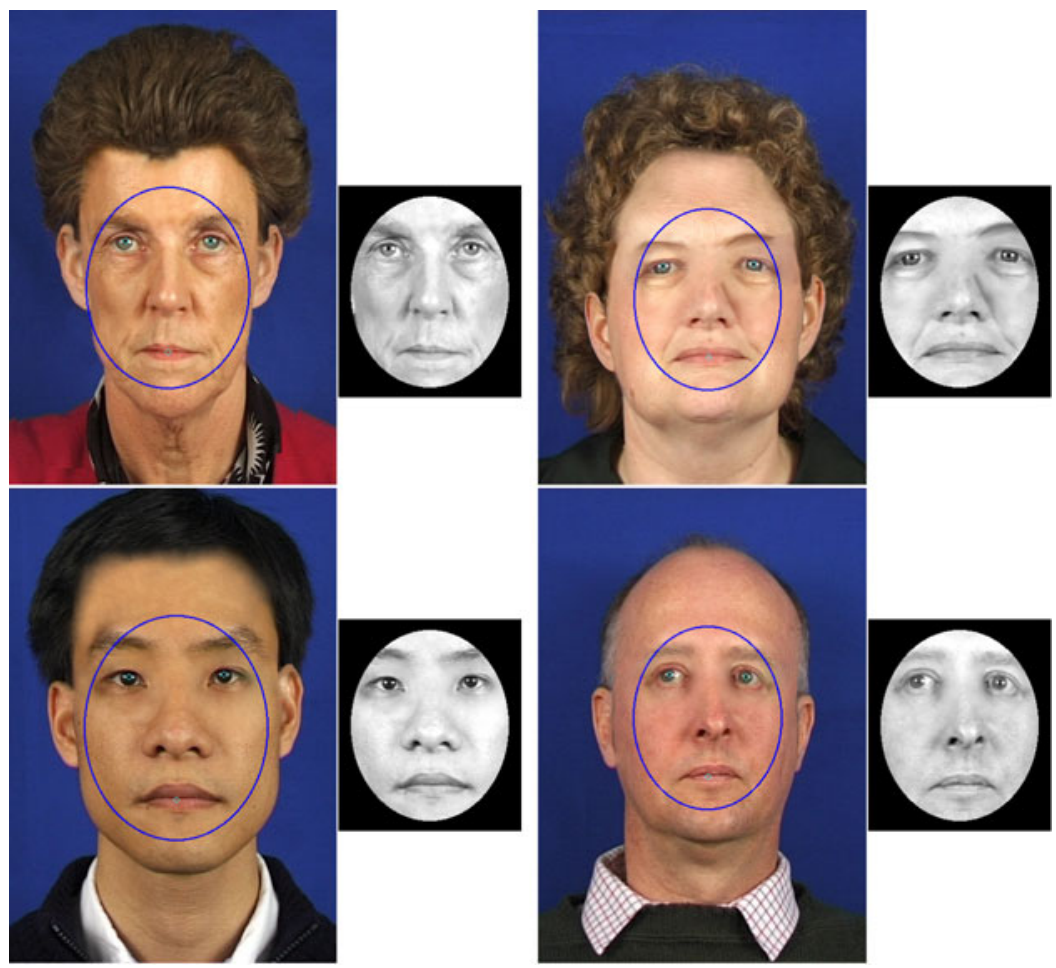

Figure 4. Examples of the geometrical standardization. In each color picture, the positions of the eyes and mouth are marked by small circles and the derived oval shapes are depicted in blue. The corresponding scaled oval grayscale images are presented next to the color pictures.

the $1 \%$ and $99 \%$ thresholds, or between 38 and 204 (Figure 3D), but this resulted in eyes that were too dark. After comparing the three contrast enhancements for a number of examples, we decided to apply the second one as a compromise between low and high contrast. So, for all faces, the grayscale values were mapped from the range between the minimum grayscale value and the $98 \%$ threshold onto the range $0-255$.

\section{Other Face Databases}

The same geometrical and optical standardization was also applied to a few other databases:

1. Max Planck Institute for Biological Cybernetics Face Database (http://faces.kyb.tuebingen.mpg.de/). This database contains seven color views, at different rotation angles, of 200 heads, of which the hair was removed by some means of preprocessing.

2. Ekman's Pictures of Facial Affect (www.paulekman .com/research.html). This collection consists of 110 grayscale images of 14 different persons, each showing several emotional expressions, such as neutral, happy, angry, and so on.

3. BioID Face Database (www.bioid.com/downloads/ facedb/index.php). This data set consists of 1,521 grayscale images of 23 different persons, featuring a large variety of poses, face sizes, illumination, and backgrounds.

It was beyond the scope of the present study to develop a robust automatic detection technique for these databases as well. Therefore, we defined the positions of the eyes and mouth manually in a subset of each database: For Database 1, only the frontal views were considered; for Database 2 , only the neutral and happy facial expressions were selected as the two extreme cases; for Database 3, only frontal views of persons with neutral facial expression and without glasses and beard/moustache were taken. The same average oval mask obtained from the XM2VTSDB was also used for these databases.

\section{RESULTS}

Some typical results of the geometrical standardization for the XM2VTSDB are depicted in Figure 4. Large differences in hair style, hair color, skin color, and head size are present in the original pictures. Because these differences are fully eliminated in our final oval grayscale images, this figure presents a strong justification for the application of an oval extraction of the faces.

The mean width and height of the oval mask of all considered faces was $185.64 \pm 11.96$ pixels and $228.7 \pm 14.48$ pixels, respectively. Interesting to note is that no correlation was found between the eye-to-eye distance and the average of the left and right eye-to-mouth distances in the original (nonscaled) faces (adjusted $R^{2}=.25$ ).

Figure 5 shows a plot of the detected $(x, y)$ positions of the eyes and mouth of all 255 faces before standardization. Large scattering is observed, with a standard deviation of about 20 pixels in $x$ and about 30 pixels in $y$, averaged over the eyes and mouth. The geometrical standardization 


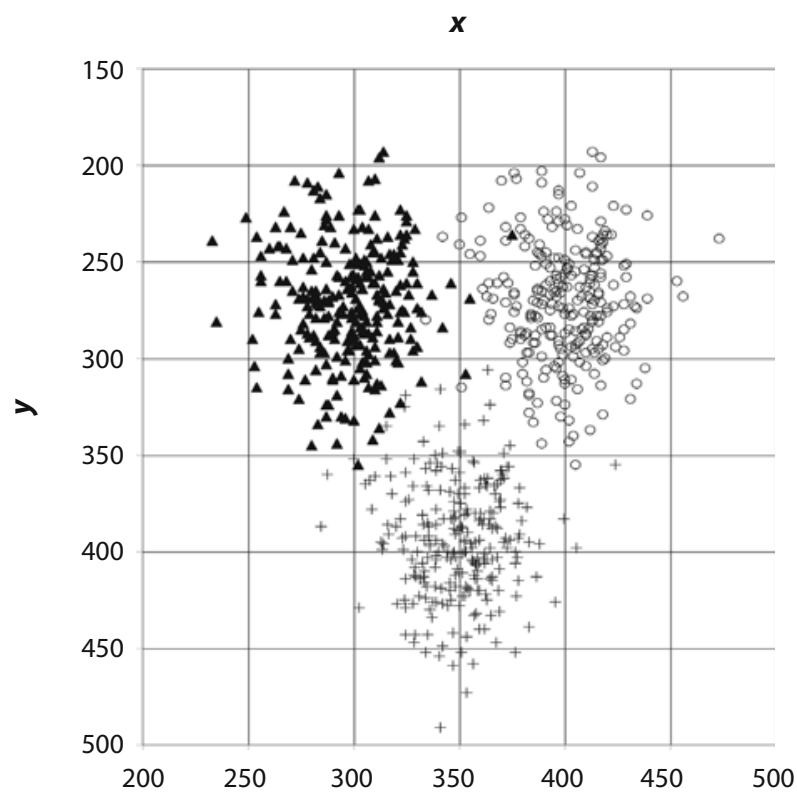

Figure 5. The $(x, y)$ positions of the left eye (triangle), right eye (circle), and mouth (plus) for all 255 original faces, before the geometrical standardization. A large scattering is observed because the faces are not centered and are not scaled to the same size.

$\boldsymbol{X}$

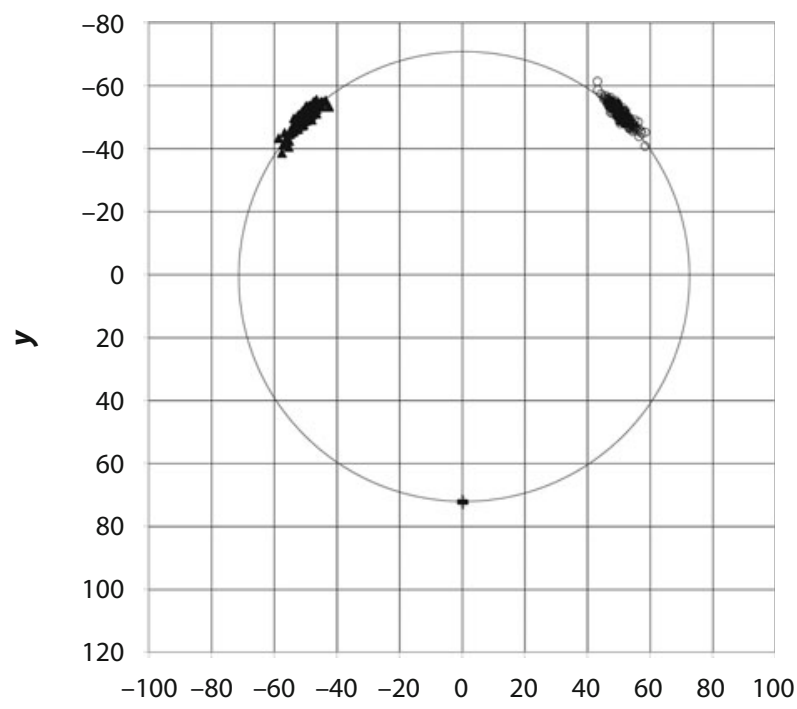

Figure 6. The $(x, y)$ positions of the left eye (triangle), right eye (circle), and mouth (plus) after geometrical standardization. In comparison with the original positions shown in Figure 5, the substantial reduction in scattering is striking. The positions are now varying around the circle on which the scaling is based.

substantially reduced the scattering, as can be observed in Figure 6: The standard deviation has now become about 2.5 pixels for both $x$ and $y$ positions of the eyes and only about 0.3 pixels for the $x$ and $y$ positions of the mouth. Ideally, the standard deviation of the mouth positions should be 0.0 . However, because we rounded off the oval center to integer positions, the observed standard deviation is very close to the theoretical value of 0.29 associated with a uniform distribution with an amplitude of 0.5 pixels. The dashed line in Figure 6 represents the circle that runs through the eyes and mouth and on which the oval shape is based.

Some results for the other three databases considered are presented in Figures A1-A4 in the Appendix.

\section{CONCLUDING REMARKS}

We developed a set of image processing steps needed to prepare stimulus materials for a face matching task in a psychological experiment. In this matching task, participants were to compare pairs of face stimuli on biological features of the faces only, in a holistic sense. The removal of image- or content-specific clues was therefore crucial. The essence of our study is a standardization of facial stimuli, and for this procedure only the positions of the eyes and mouth are required. It was demonstrated that this standardization can be successfully applied to different face databases and also to different emotional expressions and to male as well as female faces.

The proposed method proved to be simple and easy to implement, because it draws on well-established and standard image processing techniques. An automatic detection algorithm for the eyes and mouth is not a prerequisite but may be invaluable when dealing with large databases. Our detection algorithm is most probably limited to the use of the XM2VTSDB. For instance, a uniform background may not always be present (e.g., in the BioID database). However, some elements may be of general use, such as the application of a high-pass filter to detect the mouth. An advantage of the proposed method is that it is not necessary to take photos under controlled conditions (highly uniform studio shots), as was demonstrated by a successful application to the BioID database. The only requirement is that the size of the face be sufficiently large; otherwise, the quality of the scaling to the standard size may be impaired. Finally, our method is justified in a psychophysical sense because the essential facial information is retained and, at the same time, confounding factors are eliminated.

In conclusion, the proposed uniform scaling and application of an oval mask is an excellent and straightforward technique not only to standardize faces but also to retain essential featural and configurational information of the face. Finally, to be able to compare results of different studies, we propose to use the developed standardization in psychological experiments involving facial stimuli.

\section{AUTHOR NOTE}

The authors are greatly indebted to Floor Verhoeven for having taken care of most of the manual preprocessing of the picture materials. The research on which this study was based acknowledges the use of the Extended Multimodal Face Database and associated documentation, www .ee.surrey.ac.uk/Research/VSSP/xm2vtsdb/. Correspondence concerning this article should be addressed to E. H. B. M. Gronenschild, Department of Cognitive Neuropsychiatry \& Clinical Neuroscience, School for Mental Health and Neuroscience, Maastricht University, P.O. Box 616, 6200 MD Maastricht, The Netherlands (e-mail: ed.gronenschild@np.unimaas.nl). 


\section{REFERENCES}

Aylward, E. H., Park, J. E., Field, K. M., Parsons, A. C., Richards, T. L., Cramer, S. C., ET AL. (2005). Brain activation during face perception: Evidence of a developmental change. Journal of Cognitive Neuroscience, 17, 308-319.

Barton, J. J. S., Keenan, J. P., \& Bass, T. (2001). Discrimination of spatial relations and features in faces: Effects of inversion and viewing duration. British Journal of Psychology, 92, 527-549.

Bokde, A. L., Dong, W., Born, C., Leinsinger, G., Meindl, T., TeiPEL, S. J., ET AL. (2005). Task difficulty in a simultaneous face matching task modulates activity in face fusiform area. Cognitive Brain Research, 25, 701-710.

Bruce, V., Doyle, T., Dench, N., \& Burton, M. (1991). Remembering facial configurations. Cognition, 38, 109-144.

Buchala, S., Davey, N., Frank, R. J., Loomes, M., \& Gale, T. M. (2005). The role of global and feature based information in gender classification of faces: A comparison of human performance and computational models. International Journal of Neural Systems, 15, 121-128.

Dalal, A. B., \& PhadKe, S. R. (2007). Morphometric analysis of face in dysmorphology. Computer Methods \& Programs in Biomedicine, 85, $165-172$.

Dubois, S., Rossion, B., Schiltz, C., Bodart, J. M., Michel, C., BRUYER, R., ET AL. (1999). Effect of familiarity on the processing of human faces. NeuroImage, 9, 278-289.

Gonzalez, R. C., \& Woods, R. E. (1992). Digital image processing. New York: Addison-Wesley.

Haralick, R. M., Sternberg, S. R., \& Zhuang, X. (1987). Image analysis using mathematical morphology. IEEE Transactions on Pattern Analysis \& Machine Intelligence, 9, 532-550.
Kanwisher, N., McDermott, J., \& Chun, M. M. (1997). The fusiform face area: A module in human extrastriate cortex specialized for face perception. Journal of Neuroscience, 17, 4302-4311.

Leinsinger, G., Born, C., Meindl, T., Bokde, A. L., Britsch, S., Lopez-BAYO, P., ET AL. (2007). Age-dependent differences in human brain activity using a face- and location-matching task: An FMRI study. Dementia \& Geriatric Cognitive Disorders, 24, 235-246.

Messer, K., Matas, J., Kittles, J., Luettin, J., \& Maitre, G. (1999). XM2VTSDB: The extended M2VTS database. In Proceedings of the Second International Conference on Audio- and Video-Based Biometric Person Authentication (AVBPA99) (pp. 72-77). Berlin: Springer.

RAKOVER, S. S. (2002). Featural vs. configurational information in faces: A conceptual and empirical analysis. British Journal of Psychology, 93, 1-30.

Surguladze, S., Russell, T., Kucharska-Pietura, K., Travis, M. J., Giampietro, V., DaVID, A. S., ET AL. (2006). A reversal of the normal pattern of parahippocampal response to neutral and fearful faces is associated with reality distortion in schizophrenia. Biological Psychiatry, 60, 423-431.

TANaKa, J. W., \& FARah, M. J. (1993). Parts and wholes in face recognition. Quarterly Journal of Experimental Psychology, 46A, 225245.

Tong, F., Nakayama, K., Moscovitch, M., Weinrib, O., \& KanWISHER, N. (2000). Response properties of the human fusiform face area. Cognitive Neuropsychology, 17, 257-279.

White, M., \& LI, J. (2006). Matching faces and expressions in pixelated and blurred photos. American Journal of Psychology, 119, 21-28.

YIN, R. K. (1969). Looking at upside-down faces. Journal of Experimental Psychology, 81, 141-145.

\section{APPENDIX}

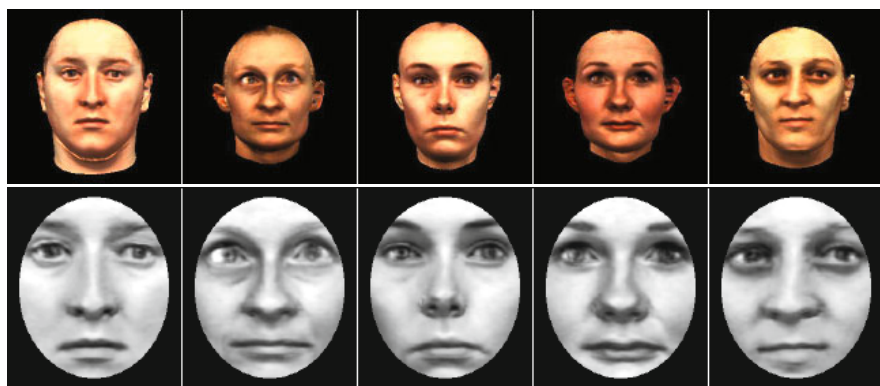

Figure A1. Some examples of the application of the standardization applied to faces from the Max Planck Institute for Biological Cybernetics Face Database.

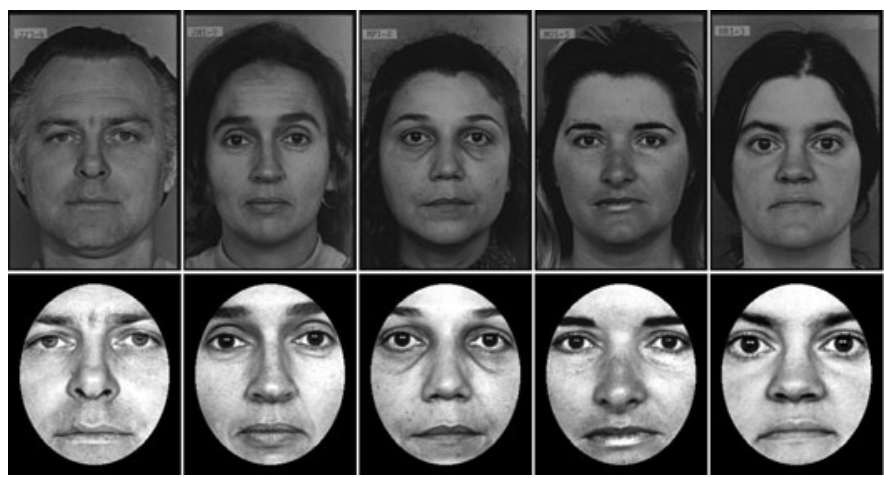

Figure A2. Some examples of the application of the standardization applied to faces with a natural expression from the Ekman's Pictures of Facial Affect database. 


\section{APPENDIX (Continued)}

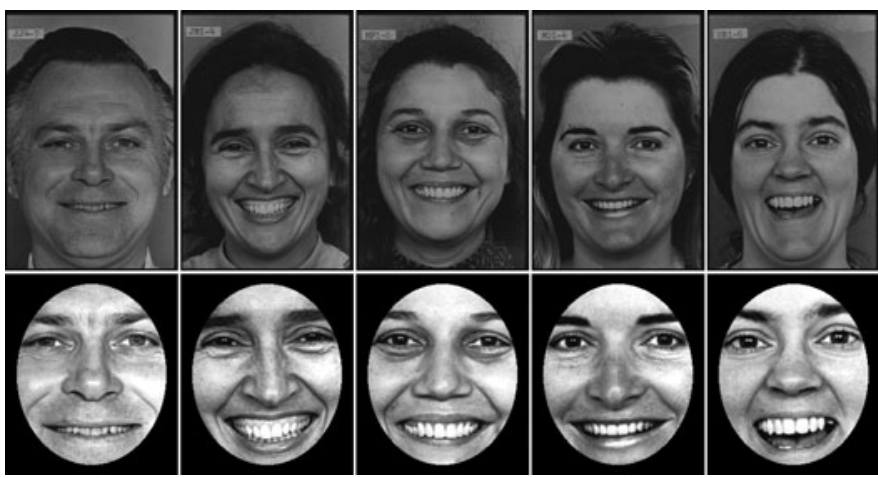

Figure A3. Some examples of the application of the standardization applied to faces with a laughing expression from the Ekman's Pictures of Facial Affect database.

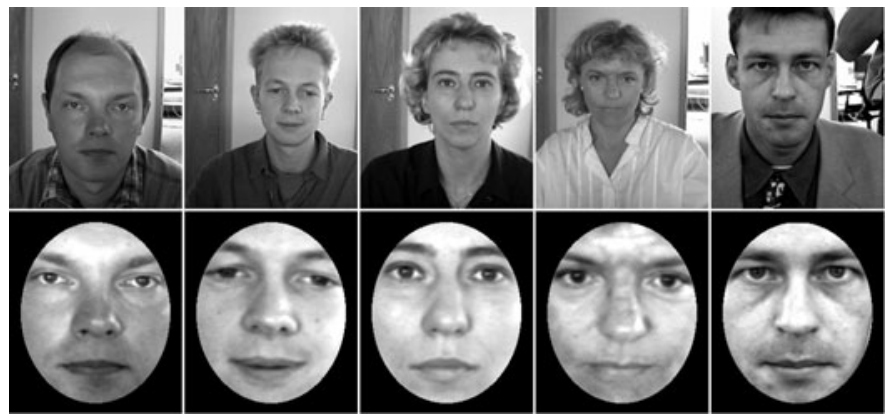

Figure A4. Some examples of the application of the standardization applied to faces from the BioID Face Database.

(Manuscript received November 26, 2008; revision accepted for publication April 10, 2009.) 